\title{
Resolution improvement in optical coherence tomography with segmented spectrum management
}

\author{
CHIH-WEI LU, MENG-TSAN TSAI, YIH-MING WANG, \\ YEAN-WOEI KIANG AND C.C. YANG* \\ Graduate Institute of Electro-Optical Engineering and Department of Electrical Engineering, National \\ Taiwan University, 1, Roosevelt Road, Section 4, Taipei, Taiwan, R. O.C. \\ (*author for correspondence: E-mail: ccy@cc.ee.ntu.edu.tw)
}

Received 15 February 2005; accepted 15 September 2005

\begin{abstract}
We demonstrate resolution improvement in an optical coherence tomography (OCT) system with multiple scans of segmented spectral bands and software signal processing. With an effective spectral full-width at half-maximum (FWHM) of $45 \mathrm{~nm}$, centered at $950 \mathrm{~nm}$, we compress the interference fringe FWHM from $31 \mu \mathrm{m}$ down to $7 \mu \mathrm{m}$, which is smaller than the theoretical limit of $8.9 \mu \mathrm{m}$ if a Gaussian spectral shape is assumed, through the combined effect of dispersion compensation and spectral shaping. With the amplitude adjustments of the OCT images of the segmented spectral bands, sidelobes can be significantly suppressed with a slight increase of the fringe envelope FWHM. With other amplitude adjustments, the envelope FWHM can be reduced to $5.6 \mu \mathrm{m}$ at the expense of significant side-lobes.
\end{abstract}

Key words: biophotonics, optical coherence tomograph, dispersion compensation, spectral shaping

\section{Introduction}

In developing optical coherence tomography (OCT), the axial resolution has been one of the key issues. Because the OCT axial resolution is inversely proportional to the spectral width of the used light source, the generation of broadband light sources has been a topic of great interest (Marks etal. 2002; Kowalevicz et al. 2002; Unterhuber et al. 2003; Wang et al. 2003). Besides the use of broadband light sources for achieving high resolution, other efforts are helpful for improving the OCT resolution. Such efforts include dispersion compensation for balancing the group delay dispersion between the reference and sample arms of an OCT system (de Boer etal. 2001; Fercher etal. 2001, 2002; Smith et al. 2002; Marks et al. 2003; Niblack etal. 2003; Zvyagin etal. 2003). Without dispersion compensation, the OCT resolution will be degraded. The other means for improving resolution is the spectral shaping technique. Spectral shaping means to modify the spectral intensity and phase distributions for controlling the time-domain behaviors. It has been widely used for modifying the pulse shape and width (Weiner and Kan'an 1998; Grote and Fouckhardt 
1999; Takeoka etal. 2001). This technique was also used for changing the width and shape of the interference fringe pattern of an OCT system. It is particularly useful when the light source spectrum is not Gaussianlike. With a non-Gaussian-like spectral profile, side-lobes in the interference fringe pattern exist. Such side-lobes will degrade the OCT resolution. A software approach, based on an iteration retrieval algorithm, has been proposed to reduce the side-lobes (Hsu et al. 2003). Also, spectral shaping effects were obtained from the spectrally filtered response of each depth profile by multiplying the Fourier transform of each individual depth profile with a spectral correction function and performing the inverse transform (Tripathi et al. 2002). Spectral shaping is quite a promising technique for improving the OCT resolution.

In this paper, we report an alternative technique for spectral management. In this technique, OCT images are first obtained with segmented light source spectra. Then, an appropriate combination of these images of different central wavelengths can result in various results of either higher axial resolution or reduced side-lobe intensity. Such a combination can be dynamically adjusted for different purposes. Also, it can be used for dispersion compensation. This technique is particularly useful for an OCT system of a large source spectral width. Although the proposed method is somewhat similar to the spectral-domain OCT in concept (Wojtkowski et al. 2004), it is quite valuable because the time-domain OCT still has certain advantages over the spectral-domain counterpart. In this paper, we discuss the OCT experimental setup for demonstrating the proposed spectral management technique in Section 2. The spectral management results are presented in Section 3. Several features of the proposed technique are discussed in Section 4. Finally, conclusions are drawn in Section 5.

\section{Experimental procedures}

The OCT system setup used for demonstrating the proposed spectral management approach is shown in Fig. 1. A super-luminescence diode (SLD) was used as the light source with the center wavelength at $950 \mathrm{~nm}$ and the bandwidth of about $70 \mathrm{~nm}$. However, because of the imperfect isolator used for protecting the SLD, the available spectrum was only $45 \mathrm{~nm}$ in full-width at half-maximum (FWHM), which corresponds to a theoretical axial resolution of $8.9 \mu \mathrm{m}$ if the spectral shape is Gaussian. In the reference arm, an optical phase delay line (OPDL) was used for group- and phase-delay modulations. To match the optical paths between the reference and sample arms, an about $25 \mathrm{~cm}$ fiber length difference is required. In this situation, the interference fringe envelope is broadened because of the dispersion mismatch. As shown in Fig. 2, the FWHM of the interference fringe envelope 




Fig. 1. Experimental setup of the OCT system.



Fig. 2. Interference fringe without dispersion compensation. The FWHM is about $100 \mu \mathrm{m}$.

is as large as $100 \mu \mathrm{m}$. In the proposed approach, we used a spatial filter, located between the lens and the galvanometer optical scanner, for gating different wavelength bands of the light source spectrum (see Fig. 3). We conducted a longitudinal scan with each spectral band. Due to dispersion, the interference fringes of different spectral bands will appear at different longitudinal positions. After properly adjusting the positions and amplitudes of the scanning images of various segmented spectral bands, their superposition leads to the results of various spectral management effects.

In our experiment, the focal length of the lens in the OPDL was $10 \mathrm{~cm}$. The grating and galvanometer optical scanner were first placed on the focal planes. With such an arrangement, the FWHM of the interference fringe envelope was $100 \mu \mathrm{m}$, as shown in Fig. 2. Here, one can observe the chirped interference fringe. To improve the system resolution, we reduce the distance between the lens and the grating to $9 \mathrm{~cm}$. With this arrangement, the FWHM of the interference fringe envelope was reduced to $31 \mu \mathrm{m}$, as shown in Fig. 4. Because dispersion is not fully compensated, this envelope width is still quite large and its shape is asymmetric. The spectral 


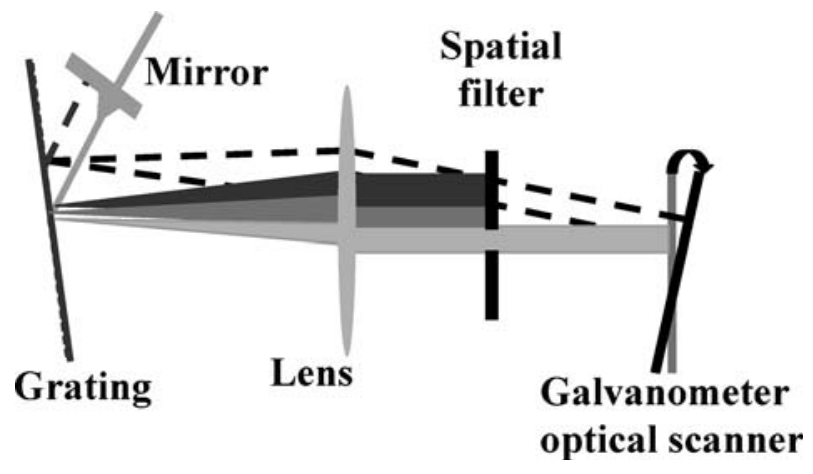

Fig. 3. The spatial filter inserted between the lens and the galvanometer optical scanner to gate different wavelength bands.

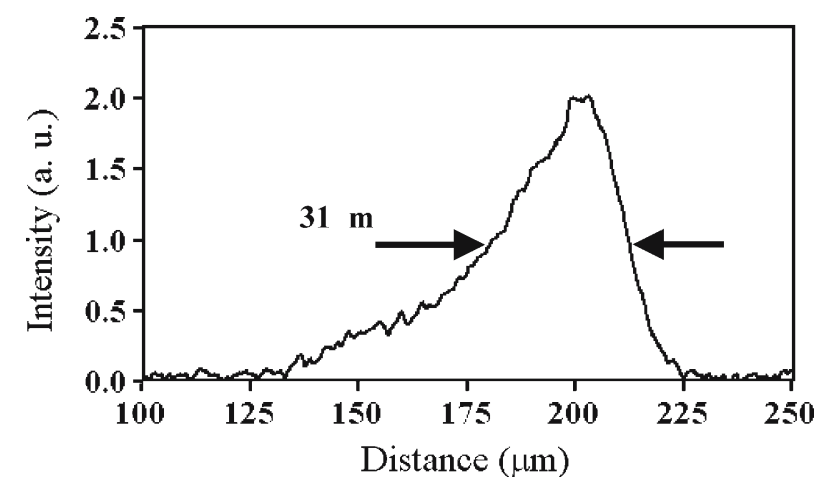

Fig. 4. Interference fringe envelope after decreasing the distance between the grating and the lens in the OPDL. The FWHM is reduced from $100 \mu \mathrm{m}$ to $31 \mu \mathrm{m}$.

segmentation is implemented by translating the 1-mm wide spatial filter in the transverse direction to the optical path. Six segmented spectral bands and hence six depth scans were obtained by translating the spatial filter with $700 \mu \mathrm{m}$ in step size. The central wavelengths of the six bands are located at 930 through $980 \mathrm{~nm}$ with $10 \mathrm{~nm}$ in step size. Each has a FWHM of about $15 \mathrm{~nm}$ and a super-Gaussian shape.

\section{Experimental results}

In Fig. 5, the interference fringe patterns from the six longitudinal scans of a glass surface with the six successive segmented spectral bands are shown. Parts (a)-(f) correspond to the results from the short to the long central wavelength. Here, one can see that the six fringe patterns are located 




Fig. 5. The six interference fringes with different segmented spectral bands.

at slightly different positions due to dispersion. The FWHMs of those interference fringe envelopes are all about $27 \mu \mathrm{m}$, which is close to the theoretical limit of the $15 \mathrm{~nm}$ FWHM in a segmented spectral band. This result shows that the dispersion effect is negligible in such a small bandwidth. It actually justifies the choice of $1 \mathrm{~mm}$ for the upper limit of the window width of the spatial filter. If the spatial filter window is too small, the available optical power in each segmented spectral band becomes too small to guarantee the sensitivity of the OCT system. Note that the modulation frequencies of the six interference fringes are also different because of their different central wavelengths.

After shifting the positions of the six interference-fringe patterns to align their envelope maxima in the horizontal axis, their summation results in a new one of a smaller envelope width, in which the dispersion mismatch has been significantly compensated. The envelope FWHM is now reduced to $7 \mu \mathrm{m}$, as shown in Fig. 6. Note that significant side-lobes exist in Fig. 6 


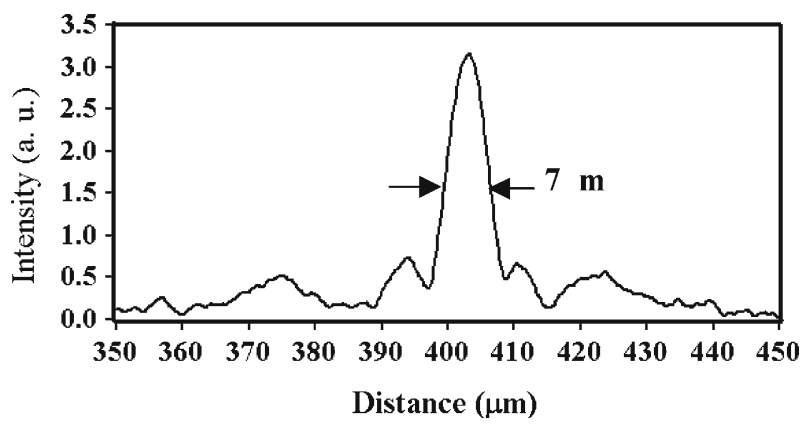

Fig. 6. Interference fringe envelope (reduced to $7 \mu \mathrm{m}$ ) after the software process.

with the intensity up to $22.6 \%$ of the main peak. The OCT resolution of $7 \mu \mathrm{m}$ is higher than the theoretical limit $(8.9 \mu \mathrm{m})$ if the spectral shape is Gaussian. The smaller envelope FWHM can be attributed to the larger effective full spectral width, which can be obtained by taking the summation of the six segmented spectral bands, because the two successive bands overlap by $5 \mathrm{~nm}$. In other words, spectral shaping has been effectively implemented. The reduced envelope FWHM was obtained at the expense of significant side-lobes.

Spectral management can also be used for side-lobe suppression. Following the same procedure as above except that the amplitudes of the six patterns in Fig. 5 are multiplied by $0.7,0.6,1,1.2,1.1$, and 0.9 (from parts (a) to (f)), respectively, before the maximum alignment and superposition, we obtain the fringe envelope in Fig. 7. Here, the FWHM is slightly increased to $7.4 \mu \mathrm{m}$. However, the side-lobes are significantly suppressed. The intensities of the side-lobes are now lower than $14.7 \%$ of the main peak.

Spectral management can lead to an even more significant envelope FWHM reduction at the expense of more significant side-lobes. Figure 8



Fig. 7. Interference fringe envelope with the side-lobes significantly suppressed after certain spectral shaping. 


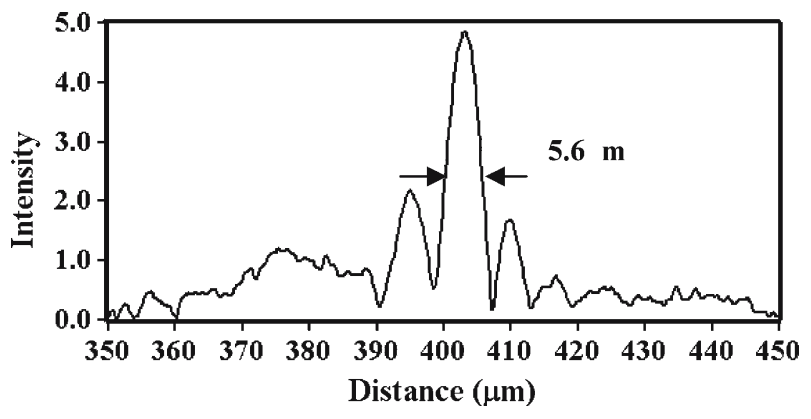

Fig. 8. Interference fringe envelope (reduced to $5.6 \mu \mathrm{m}$ ) after the spectral shaping process with another set of parameters.

shows the result by multiplying the amplitudes of parts (a)-(f) in Fig. 5 by $3,1,1,1,2.5$ and 5, respectively, before the maximum alignment and superposition. Now, the FWHM is reduced to $5.6 \mu \mathrm{m}$. However, the side-lobe intensity becomes as large as $45.3 \%$ of the main peak. Such an interference fringe envelope may not be suitable for biomedical diagnosis; however, it can be used for the scanning of a multiple-layer structure, in which only the main peak feature is needed.

\section{Discussions}

The major cost we need to pay for the system improvement with the proposed spectral management method is the longer imaging time because multiple scans of segmented spectral bands are required. However, it has several advantages as discussed in the following. First, it can be flexibly used for optimizing the effects of improving the OCT resolution or suppressing the side-lobes, depending on the particular scanning need. Second, it can also be used for dispersion compensation. The alignment of the envelope maxima of parts (a)-(f) in Fig. 5 before superposition means to compensate the dispersion mismatch between the reference and sample arms. In other words, the separations between the six interference fringes represent the group delay differences of the OCT system at various wavelengths. Figure 9 shows the relative group delay data and a curve of quadratic polynomial fitting. With the concave-upward curve, one can read the decreasing trend of normal dispersion with increasing wavelength in this OCT system. The results shown in Figs. 6-8 were obtained through the combined effects of dispersion compensation and spectral shaping.

It is noted that in concept the proposed dispersion management method is somewhat similar to the technique of spectral-domain OCT. Spectraldomain OCT has the advantages of faster scanning, higher signal-to-noise 




Fig. 9. Data of the relative group delay in the OCT system and their quadratic polynomial fit (the continuous curve).

ratio (de Boer et al. 2003), and systematical software dispersion compensation (Wojtkowski etal. 2004). However, so far it still has certain limitations, including the unavailability of arrayed detectors beyond $1 \mu \mathrm{m}$ in wavelength, the sensitivity to sample motion, and the fast drop of signal-tonoise ratio when the path lengths of the sample and reference arms are not exactly matched (Leitgeb et al. 2003). Time-domain OCT with the proposed dispersion compensation method still deserves exploration.

\section{Conclusions}

In summary, we have demonstrated an alternative approach for spectral management in an OCT system with multiple scans of segmented spectral bands and software signal processing. With an effective spectral FWHM of $45 \mathrm{~nm}$, centered at $950 \mathrm{~nm}$, we compressed the interference fringe FWHM from $31 \mu \mathrm{m}$ down to $7 \mu \mathrm{m}$, which was smaller than the theoretical limit of $8.9 \mu \mathrm{m}$ if a Gaussian spectral shape was assumed, through the combined effect of dispersion compensation and spectral management. With the amplitude adjustments of the OCT images of the segmented spectral bands, side-lobes could be significantly suppressed with slight degradation of axial resolution. With other amplitude adjustments, the fringe-envelope FWHM was reduced to $5.6 \mu \mathrm{m}$ at the expense of more significant side-lobes.

\section{Acknowledgement}

This research was supported by National Health Research Institute, The Republic of China, under the grant of NHRI-EX93-9220EI. 


\section{References}

de Boer, J.F., C.E. Saxer and J.S. Nelson. Appl. Opt. 40 5787, 2001.

de Boer, J.F., B. Cense, B.H. Park, M.C. Pierce, G.J. Tearney and B.E. Bouma. Opt. Lett. 282067 , 2003.

Fercher, A.F., C.K. Hitzenberger, M. Sticker, R. Zawadzki, B. Karamata and T. Lasser. Opt. Exp. 9 610, 2001

Fercher, A.F., C.K. Hitzenberger, M. Sticker, R. Zawadzki, B. Karamata and T. Lasser. Opt. Comm. $20467,2002$.

Grote, R. and H. Fouckhardt. Opt. Exp. 8 328, 1999.

Hsu, I.J., C.W. Sun, C.W. Lu, C.C. Yang, C.P. Chiang and C.W. Lin. Appl. Opt. 42 227, 2003.

Kowalevicz, A.M., T. Ko, I. Hartl and J.G. Fujimoto. Opt. Exp. 10 349, 2002.

Leitgeb, R., C.K. Hitzenberger and A.F. Fercher. Opt. Exp. 11 889, 2003.

Marks, D.L., A.L. Oldenburg and J.J. Reynolds. Opt. Lett. 27 2010, 2002.

Marks, D.L., A.L. Oldenburg, J.J. Reynolds and S.A. Boppart. Appl. Opt. 42 204, 2003.

Niblack, W.K., J.O. Schenk, B. Liu and M.E. Brezinski. Appl. Opt. 42 4115, 2003.

Smith, E.D.J., A.V. Zvyagin and D.D. Sampson. Opt. Lett. 27 1998, 2002.

Takeoka, M., D. Fujishima and F. Kannari. Opt. Lett. 26 1592, 2001.

Tripathi, R., N. Nassif, J.S. Nelson, B.H. Park and J.F. de Boer. Opt. Lett. 27 406, 2002.

Unterhuber, A., B. Povazay, B. Hermann, H. Sattmann and W. Drexler. Opt. Lett. 28 905, 2003.

Wang, Y., Y. Zhao, J.S. Nelson and Z. Chen. Opt. Lett. 28 182, 2003.

Weiner, A.M. and A.M. Kan'an. IEEE J. Select. Top. Quan. Electron. 4 317, 1998.

Wojtkowski, M., V.J. Srinivasan, T.H. Ko, J.G. Fujimoto, A. Kowalczyk and J.S. Duker. Opt. Exp. 12 2404, 2004

Zvyagin, A.V., E.D.J. Smith and D.D. Sampson. J. Opt. Soc. Am. A 20 333, 2003. 